\title{
The Schizotypal Ambivalence Scale as a Marker of Schizotypy
}

By: Monica C. Mann MA, Amanda G. Vaughn BA, Neus Barrantes-Vidal, PhD, Michael L. Raulin,PhD and Thomas R. Kwapil PhD†

Mann, M.C., Vaughn, A.G., Barrantes-Vidal, N., Raulin, M.L., \& Kwapil, T.R. (2008). The Schizotypal Ambivalence Scale as a marker of schizotypy. Journal of Nervous and Mental Disease, 196, 399-404. http://dx.doi.org/10.1097/NMD.0b013e3181710900

Made available courtesy of Lippincott, Williams \& Wilkins: http://www.lww.com

\author{
*** Reprinted with permission. No further reproduction is authorized without written \\ permission from Lippincott, Williams \& Wilkins. This version of the document is not the final \\ published version. Figures and/or pictures may be missing from this format of the \\ document. ***
}

\begin{abstract}
:
The present study examined the psychometric properties of the Schizotypal Ambivalence Scale (SAS) in a sample of 1798 young adults. The study also investigated the concurrent validity of the measure for identifying schizophrenic-like symptoms in a sample of 43 high scorers on the scale and 43 control participants. Previous findings indicated that high scores on the SAS were associated with schizophrenia-spectrum pathology in a sample of schizotypic young adults selected with other measures. However, this is the first study to assess schizophrenic-like psychopathology in a sample selected using the SAS. The SAS has good internal consistency (coefficient [alpha] $=0.84$ ) and test-retest reliability (intraclass correlation $=0.74$ across 9 weeks). As hypothesized, the ambivalence group exceeded the control group on interview ratings of schizotypal, schizoid, paranoid, psychotic-like, and negative symptoms, as well as exhibiting poorer overall functioning. The SAS seems to be a promising measure of schizotypy in young adults.
\end{abstract}

Keywords: schizotypal | schizotypal ambivalence | schizophrenia | psychology

\section{Article:}

The present study examined the psychometric properties and concurrent validity of the Schizotypal Ambivalence Scale (SAS; Raulin, 1986) for identifying schizophrenic-like symptoms and other forms of psychopathology in a sample of college students. The study built 
upon (Kwapil et al., 2002) preliminary findings for the scale by providing norms and reliability estimates for a large college student sample, examining the relationship of the scale with other psychometric indicators of schizotypy, and examining the concurrent validity of the scale for identifying schizotypy.

Ambivalence, Schizotypy, and Schizophrenia

The term ambivalence was coined by Bleuler (1911/1950) to describe the "tendency of the schizophrenic psyche to endow the most diverse psychisms with both a positive and negative indicator at 1 and the same time” (p. 53) (e.g., simultaneously experiencing intense love and hatred for a person). Bleuler hypothesized that ambivalence was 1 of 4 fundamental symptoms of schizophrenia—symptoms that he argued were always present in the disorder. Likewise, Meehl (1962) described ambivalence as 1 of the 4 core symptoms of schizotypy (the latent personality organization found in those individuals at genetic risk for schizophrenia). However, in later writings, Meehl $(1989,1990)$ assigned ambivalence a secondary role as a potentiating factor in schizotypy - that is, a factor that increases risk for deterioration in those people who have specific risk factors for schizophrenia.

Despite the prominent role of ambivalence in both Bleuler's formulation of schizophrenia and Meehl's initial model of schizotypy, the construct received little attention from developmental and experimental psychopathologists studying schizotypy and schizophrenia. The lack of research resulted in large part from a failure to adequately operationalize the construct, as well as a gradual modification of the construct by psychoanalytic theorists studying borderline personality (e.g., Kernberg, 1977; see Raulin and Brenner, 1993, for a review). However, recent studies have attempted to operationalize ambivalence and assess its relationship with schizotypy and schizophrenia, defining ambivalence based largely on Bleuler and Meehl's formulations.

\section{Psychometric Assessment of Ambivalence}

Several studies have examined the relationship of ambivalence with schizophrenic-like psychopathology in clinical and nonclinical samples. Raulin (1984) developed the 45-item Intense Ambivalence Scale as part of a larger effort to develop psychometric inventories for screening adolescents and young adults at risk for schizophrenia. This initial scale was based on the description of intense ambivalence provided by Meehl (1964) in his Checklist of Schizotypic Signs. Raulin (1984) found that patients with schizophrenia scored significantly higher on the scale than nonpatient control participants, but not significantly higher than a group of heterogeneous outpatient clinic clients. Furthermore, depressed inpatients scored significantly higher on the scale than patients with schizophrenia. Kwapil et al. (2000) examined the predictive validity of the Intense Ambivalence Scale in a subset of schizotypic and control 
participants from Chapman et al.'s (1994) 10-year longitudinal study. The schizotypic participants were identified by deviantly high scores on the perceptual aberration (Chapman et al., 1978) and the magical ideation (Eckblad and Chapman, 1983) scales. Elevated scores on the Intense Ambivalence Scale predicted psychotic-like, schizotypal, and depressive symptoms, substance abuse, and the development of psychotic illness at the 10-year follow-up assessment, even after the removal of variance for membership in the schizotypy and control groups.

Because the original Intense Ambivalence Scale proved to be especially sensitive to depressive symptomatology, instead of specific to the ambivalence thought to be characteristic of schizophrenia and schizotypy, Raulin (1986) developed a second ambivalence scale referred to as the SAS. This scale included a subset of items from the original scale that differentiated schizophrenic patients from controls and from psychotically depressed patients. Kwapil et al. (2002) provided a preliminary examination of the psychometric properties and concurrent validity of the SAS. They conducted diagnostic interviews with a sample of 131 college students identified by deviantly high scores on the perceptual aberration and magical ideation scales and control participants. In this sample, elevated scores on the SAS were associated with schizotypal, schizoid, and paranoid symptoms, as well as poorer overall functioning (after the removal of variance associated with perceptual aberration/magical ideation and control group membership). Unlike the findings for the Intense Ambivalence Scale, the revised scale was not associated with elevated rates of mood disorders or ratings of substance use or abuse. These findings suggested that the SAS might be a more promising measure for identifying ambivalence that is specifically associated with schizophrenia and schizotypy. However, the findings of Kwapil et al. (2002) were limited by the fact that the participants were selected based upon scores on other schizotypy questionnaires.

Goals and Hypotheses of the Present Study

The present study extended the preliminary findings of Kwapil et al. (2002) by examining the psychometric properties of the SAS, including internal consistency and test-retest reliability in a large sample of college students. The study also assessed the concurrent validity of the SAS in a sample of high scorers on the scale and control participants. Unlike Kwapil et al. (2002), the present study examined concurrent validity of the scale in a new sample of ambivalence and control participants selected solely on scores on the SAS. It was hypothesized that participants selected based on high scores on the SAS would exhibit elevated rates of schizophrenic-like symptoms and poorer overall adjustment relative to control participants. 


\section{METHODS}

\section{Participants}

Screening packets were completed by 1798 college students enrolled in General Psychology courses at the University of North Carolina at Greensboro over the course of 5 semesters. These participants included 1057 white females, 354 African American females, 322 white males, and 65 African American males. A subset of 166 participants (75 white females, 33 African American females, 46 white males, and 12 African American males) volunteered to be retested on the SAS after an 8- to 11 -week interval $(M=9.1$ week, $S D=0.9)$ to examine test-retest reliability of the scale. The reassessed participants did not differ from the remaining sample on mean score on the SAS at the initial assessment.

Eighty-six participants selected from the latter 3 semesters of mass screening were administered a structured diagnostic interview. The interview participants included 43 individuals who received standard scores of at least +1.96 on the SAS, and 43 control participants who had standard scores of less than +0.5 on the scale [the selection criteria were consistent with the recommendations of Chapman et al. (1994)]. Sixteen additional participants (10 from the ambivalence and 6 from the control groups) declined to participate in the study or failed to attend scheduled appointments. Table 1 lists the demographic characteristics of the interview participants. The groups did not differ on any of the demographic characteristics.

Table 1

TABLE 1. Demographic Characteristics of the Interviewed Participants

\begin{tabular}{|c|c|c|c|c|}
\hline & \multicolumn{4}{|c|}{ Group } \\
\hline & \multicolumn{2}{|c|}{$\begin{array}{c}\text { Schizotypal } \\
\text { Ambivalence } \\
(n=43)\end{array}$} & \multicolumn{2}{|c|}{$\begin{array}{c}\text { Control } \\
(n=43)\end{array}$} \\
\hline & Mean & $S D$ & Mean & $S D$ \\
\hline Age & 19.6 & 2.6 & 19.0 & 0.9 \\
\hline Years of education & 12.3 & 0.6 & 12.3 & 0.8 \\
\hline Parental social position & 33.0 & 11.0 & 29.1 & 12.3 \\
\hline Female $(\%)$ & 65 & & 61 & \\
\hline African American (\%) & 23 & & 28 & \\
\hline
\end{tabular}

Social position is a weighted composite rating of educational and occupational functioning for which higher scores indicate poorer accomplishment. Each participant's parental social position is based upon the parent with the lowest score (highest social position). 
Materials and Procedure

Mass-Screening Packet

The screening packet contained the SAS intermixed with the perceptual aberration, magical ideation, revised social anhedonia (Eckblad et al., 1982), and physical anhedonia scales (Chapman et al., 1976), and a 13-item infrequency scale (Chapman and Chapman, 1986). The students completed the questionnaires in group-testing sessions that lasted approximately 90 minutes and they received course credit for their participation. Participants who received a score of 3 or above on the infrequency scale were omitted from the study.

\section{Schizotypy Scales}

The SAS contains 19 true-false items that emphasize the simultaneous experience of contradictory emotions or the rapid and almost random change of emotions back and forth over time. The perceptual aberration scale contains 35 items that assess mild schizophrenic-like perceptual and body-image distortions, whereas the magical ideation scale contains 30 items that assess belief in experiences that are generally considered implausible or invalid. The revised social anhedonia scale contains 40 items that tap schizoid asociality and social disinterest. The physical anhedonia scale consists of 61 items that assess deficits in esthetic and sensory gratification. Coefficient [alpha] was greater than 0.80 for each of the measures in the present sample.

Structured Diagnostic Interview

The interview contained the portions of the structured clinical interview for DSM-IV (First et al., 1995) that assess mood disorders, substance use, and demographic information. Mood episodes were coded as present/absent, whereas substance use and impairment were coded using the quantitative rating system described in Kwapil (1996). The modules of the International Personality Disorders Examination (World Health Organization, 1995) that assess schizoid, paranoid, and schizotypal personality disorders were also included. The Wisconsin manual for assessing psychotic-like experiences (Chapman and Chapman, 1980; Kwapil et al., 1999) was used to rate psychotic symptoms across a broad range of clinical and subclinical deviancy. The negative symptom manual (Kwapil and Dickerson, unpublished data, 2001), which provides a companion rating system to the Wisconsin manual, was used to quantify negative symptoms of schizophrenia across a range of clinical and subclinical deviances. Each participant's overall functioning was rated by the interviewer using the global adjustment scale (GAS; Endicott et al., 1976), which indicates current functioning with a range from extreme psychopathology to 
superior adjustment. Familial socioeconomic status was rated using Hollingshead's (1957) 2factor index of social position. The index of social position is a weighted composite measure of occupation and education (with higher scores indicating lower social position).

A licensed clinical psychologist and 2 advanced undergraduate students with extensive training conducted and scored the interviews. The interviewers were unaware of the participants' group membership. Although interrater reliability was not assessed on this sample, reliability data from our laboratory are available on several of these measures. Interrater reliability was 0.89 for the Wisconsin manual, 0.94 for the negative symptom manual, and 0.87 for the GAS. The diagnostic interviews lasted approximately 2 hours and were audiotaped. Students received course credit or payment for their participation.

\section{RESULTS}

Psychometric Properties of the SAS

The psychometric properties of the SAS were examined for the 1798 participants in the massscreening sample (total sample: $\mathrm{M}=6.51, \mathrm{SD}=4.36$, range $=0-19$, coefficient [alpha] $=0.84$ ). The distribution of scores was positively skewed and flattened (skew $=0.52$; kurtosis $=-0.47$ ). The positive skew of the distribution resulted from the inclusion of items of relatively low endorsement to create a scale that was more discriminating at the high end of the distribution. Item-scale correlations for the SAS ranged from 0.43 to 0.57 , and item endorsement rates ranged from 0.16 to 0.69 . Note that despite the initial selection of items with a low rate of endorsement, 3 of the items had endorsement rates of greater than $50 \%$ in the present sample. The SAS correlated 0.50 with the perceptual aberration scale, 0.47 with the magical ideation scale, 0.45 with the revised social anhedonia scale, and 0.18 with the physical anhedonia scale (all correlations, $\mathrm{p}<0.001$ ). The intraclass correlation for the 9-week test-retest reliability was 0.74 , $\mathrm{p}<0.001$.

A $2 \times 2$ (gender $\times$ ethnicity) ANOVA was computed on the total SAS score. Neither the main effect for gender, $F(1,1794)=0.26$, Cohen's $d=0.02$, nor the ethnicity $\times$ gender interaction, $\mathrm{F}(1,1794)=0.34$, was significant. The main effect for ethnicity, was significant, $\mathrm{F}(1,1794)=$ 8.93, $\mathrm{p}<0.01, \mathrm{~d}=0.17$, with African Americans $(\mathrm{M}=7.09$, $\mathrm{SD}=4.20)$ exceeding whites $(\mathrm{M}=$ $6.34, \mathrm{SD}=4.40)$. However, these findings should be interpreted cautiously given the sample size and small effect size. 


\section{Interview Study}

Table 2 presents comparisons of the schizotypal ambivalence and control groups on measures of psychopathology and overall adjustment. The ambivalence group exceeded the control group on ratings of psychotic-like, negative, schizotypal, schizoid, and paranoid symptoms, and evidenced poorer overall adjustment as assessed by the GAS. The ambivalence group demonstrated a trend toward more drug use, but the groups did not differ on ratings of alcohol use or on alcohol or drug impairment (the mean ratings reflected minimal use and impairment in both groups). Note that results were not presented separately by gender and ethnic group in Table 2 because inclusion of gender and ethnicity did not produce any substantive changes in the results. All of the t-tests comparisons were recomputed as multiple regression analyses with gender and ethnicity entered at the first step and group membership entered at the second step. The removal of the variance associated with gender and ethnicity did not change the significance of any of the group comparisons reported in Table 2. The groups did not differ significantly on the proportion of participants with major depressive episodes (Fisher exact test $=0.20$ ), and none of the participants had experienced a manic episode.

Table 2

TABLE 2. Comparison of the Schizotypal Ambivalence and Control Groups on Interview Measures of Psychopathology and Adjustment

\begin{tabular}{|c|c|c|c|c|}
\hline & $\begin{array}{l}\text { Schizotypal Ambivalence } \\
\qquad(n=43)\end{array}$ & $\begin{array}{l}\text { Control } \\
(n=43)\end{array}$ & $\begin{array}{c}t \text { Value } \\
(d f=84)\end{array}$ & Cohen's $d$ \\
\hline Psychotic-like experiences & $1.2(2.1)$ & $0.4(1.2)$ & $2.31^{*}$ & 0.50 \\
\hline Negative symptom manual & $4.4(4.3)$ & $0.7(1.2)$ & $5.42^{* \omega}$ & 1.17 \\
\hline \multicolumn{5}{|l|}{ IPDE dimensional scores } \\
\hline Schizotypal & $1.4(2.0)$ & $0.4(0.8)$ & $3.11^{\text {**** }}$ & 0.67 \\
\hline Schizoid & $0.8(1.3)$ & $0.2(0.4)$ & $2.97^{* * *}$ & 0.64 \\
\hline Paranoid & $1.4(2.4)$ & $0.2(0.6)$ & $3.27^{\phi * *}$ & 0.70 \\
\hline Global assessment scale & $71.5(9.0)$ & $81.7(6.6)$ & $6.00^{* *}$ & 1.29 \\
\hline Major depressive episodes (\%) & 18.6 & 7.0 & NS & \\
\hline Manic episodes (\%) & 0.0 & 0.0 & NS & \\
\hline Alcohol use & $2.2(4.2)$ & $1.2(2.3)$ & 1.33 & 0.29 \\
\hline Alcohol impaiment & $0.5(0.7)$ & $0.4(0.7)$ & 0.49 & 0.10 \\
\hline Drug use & $0.5(1.1)$ & $0.2(0.6)$ & 1.70 & 0.37 \\
\hline Drug impairment & $0.3(0.6)$ & $0.1(0.3)$ & 1.55 & 0.35 \\
\hline
\end{tabular}

None of the participants met criteria for a psychotic disorder at the time of the assessment. However, 1 schizotypal ambivalence group member qualified for a diagnosis of paranoid personality disorder. None of the other participants met criteria for a schizophrenia-spectrum disorder. 


\section{DISCUSSION}

In his chapter entitled, “The Fundamental Symptoms,” Bleuler (1911/1950) argued that "ambivalence is such an immediate consequence of the schizophrenic association disturbance that its complete absence appears highly improbable” (p. 53). Fifty years later, Meehl (1962) incorporated ambivalence as one of the core features of his model of schizotypy. However, aside from these notable inclusions, and the work by Raulin et al., the construct of ambivalence has been largely ignored in the experimental and developmental psychopathology literature. A recent literature search produced approximately 2500 citations containing the term ambivalence. However, only about 100 of these publications involved schizophrenia and related conditions and fewer than 1 dozen involved empirical studies of ambivalence in schizophrenic or schizotypic individuals. This raises the obvious questions of what did 2 of the preeminent thinkers in psychopathology find so compelling about the construct and why has it been so readily dismissed by the majority of psychopathology researchers? In other words, how do we reconcile the theoretical emphasis with the relative empirical disinterest?

Bleuler (1911/1950) recognized 3 subtypes of ambivalence (ambivalence of affect, will, and intellect). The ambivalence measured by the SAS is what Bleuler call ambivalence of affect. Bleuler's ambivalence of intellect is very close to our current concept of thought disorder. Bleuler viewed disordered thoughts and disordered emotions as similar phenomena, a view that is consistent with some current theories in neuroscience (e.g., Minsky, 2006). Bleuler argued that each of these subtypes of ambivalence resulted from a disruption of associative threads, suggesting that "the schizophrenic, with his weakened associative linkings does not necessarily bring the different aspects of a problem together” (p 374). Meehl (1989) viewed hypohedonia and ambivalence as resulting from a combination of neurodevelopmental deficits and aversive drift (resulting in large part from negative social learning). The everyday experience of ambivalence in healthy individuals (experienced as indecision or relatively normal vacillation in feelings and interests) probably has little in common with the clinical ambivalence first described by Bleuler. Bleuler's description of ambivalence is so dramatic that it borders on thought disorder and appears well outside the range of normal experience. However, the ubiquitous nature of normal ambivalent tendencies has likely contributed to the difficulty of rigorously identifying its clinical counterpart. As noted above, the problem of adequately operationalizing ambivalence has clearly worked against its formal study. Raulin recognized and attempted to address these shortcomings with the Intense Ambivalence Scale and later the SAS. Both of these measures seem to be psychometrically sound measures of ambivalence; however, the SAS seems to be 
especially promising for the assessment of the type of ambivalence that is related to schizophrenia and schizotypy.

The present findings indicate that psychometrically identified ambivalence is associated with schizophrenic-like psychopathology in a nonclinical sample of young adults [over-and-above the preliminary work by Kwapil et al. (2000)]. Furthermore, unlike the earlier findings for the Intense Ambivalence Scale, the SAS was not broadly associated with psychopathology, but much more strongly related to schizophrenic-like impairment. Specifically, the effect sizes were in the medium to large range for measures of schizophrenia-spectrum psychopathology and impairment, but only in the small range for other forms of psychopathology.

The last 20 years have seen an increasing focus on Bleuler's notion that negative symptoms of schizophrenia represent the fundamental disturbance in the disorder (e.g., Andreasen, 1990; Blanchard and Cohen, 2006; Faraone et al., 2001; Keefe et al., 1992; Lenzenweger and Dworkin, 1996). Notably, the SAS group had elevated scores on measures of negative symptoms (e.g., the negative symptom manual and the schizoid dimensional score). Interestingly, the SAS correlated moderately with the perceptual aberration and magical ideation scales (measures of positive symptom schizotypy) and the revised social anhedonia scale (a measure of negative symptom schizotypy). All 3 of these screening measures were found to identify risk for the development of schizophrenia-spectrum disorders in nonpsychotic college students and the combination of the magical ideation scale and revised social anhedonia scales seemed to be an especially potent predictor of psychotic illness (Chapman et al., 1994; Kwapil, 1998). The SAS had only a modest correlation with the physical anhedonia scale-a measure that has not been an effective predictor of the development of spectrum disorders in college samples. The present findings indicate that ambivalence tapped by the SAS is associated with both positive and negative schizotypy. However, future investigations should examine the extent to which ambivalence may be better conceptualized as a separate dimension of schizotypy, as well as the extent to which it maps onto other putative dimensions of the construct (e.g., Claridge et al., 1996 social anxiety/cognitive disorganization factor).

Given the solid psychometric properties of the scale and its correlations with proven psychometric inventories, it seems that the 19-item SAS provides a brief and effective method of initial screening for schizotypy. Obviously, prospective studies are required to determine the predictive validity of the SAS. Furthermore, the specificity of the scale will need more empirical evaluation. Specifically, future studies should examine the relationship of the measure with borderline personality traits, given the central role that ambivalence plays in formulation of this disorder. 
The employment of the SAS implicitly raises questions regarding the utility of the psychometric high-risk method for identifying individuals at risk for schizophrenia-spectrum disorders and for enhancing our understanding of the etiology and development of such disorders. One line of argument suggests that psychometric approaches may not be effective in the study of low baserate conditions such as schizophrenia. However, several lines of evidence suggest that the psychometric screening approach is a viable research method (e.g., Chapman et al., 1995; Edell, 1995; Fernandes and Miller, 1995; Lenzenweger, 1998). The study of schizotypy (and the etiology and development of schizophrenia) is hampered by the lack of a gold standard (other than the development of full-blown schizophrenia). Although Lenzenweger et al. (2003) caution that psychometric screening inventories identify an admixture of schizotypes and nonschizotypes, these measures seem to identify meaningful variance related to schizotypy. Furthermore, these measures work especially well in conjunction with interview and laboratory measures of schizotypy and appear to be promising adjuncts for use in high-risk samples identified by consanguinity or clinical features.

The SAS provides a reliable and brief self-report inventory that seems promising as a research measure of 1 facet of schizotypy and schizophrenia. The questionnaire is not presently recommended for applied use because of the lack of research conducted with clinical samples. The results of the present study justify further use of the measure including development of norms with different demographic groups and the assessment of the predictive validity of the questionnaire. Furthermore, the present findings, in conjunction with findings for the Intense Ambivalence Scale, suggest that further study of the construct of ambivalence and its relationship to psychopathology is warranted.

\section{References:}

Andreasen NC (1990) Positive and negative symptoms: Historical and conceptual aspects. Mod Probl Pharmacopsychiatry. 24:1-42.

Blanchard JJ, Cohen AS (2006) The structure of negative symptoms within schizophrenia: Implications for assessment. Schizophr Bull. 32:238-245.

Bleuler EP (1950) Dementia Praecox or the Group of Schizophrenias (Zinkin J, Trans.). New York: International Universities Press (original work published in 1911). 
Chapman LJ, Chapman JP (1980) Scales for rating psychotic and psychotic-like experiences as continua. Schizophr Bull. 6:476-489.

Chapman LJ, Chapman JP (1986) Infrequency Scale. Unpublished scale available from: TR Kwapil, Department of Psychology, University of North Carolina at Greensboro, Greensboro, NC 27402.

Chapman LJ, Chapman JP, Kwapil TR (1995) Scales for the measurement of schizotypy. In A Raine, T Lencz, S Mednick (Eds), Schizotypal Personality Disorder. Cambridge, England: Cambridge University Press.

Chapman LJ, Chapman JP, Kwapil TR, Eckblad M, Zinser MC (1994) Putatively psychosisprone subjects 10 years later. J Abnorm Psychol. 103:171-183.

Chapman LJ, Chapman JP, Raulin ML (1976) Scales for physical and social anhedonia. J Abnorm Psychol. 85:374-382.

Chapman LJ, Chapman JP, Raulin ML (1978) Body image aberration in schizophrenia. J Abnorm Psychol. 87:399-407.

Claridge G, McCreery C, Mason O, Bentall R, Boyle G, Slade P, Popplewell D (1996) The factor structure of "schizotypal” traits: A large replication study. Br J Clin Psychol. 35:103-115.

Eckblad M, Chapman LJ (1983) Magical ideation as an indicator of schizotypy. J Consult Clin Psychol. 51:215-225.

Eckblad M, Chapman LJ, Chapman JP, Mishlove M (1982) The Revised Social Anhedonia Scale. Unpublished scale available from: TR Kwapil, Department of Psychology, University of North Carolina at Greensboro, Greensboro, NC 27402. 
Edell WS (1995) The Wisconsin psychosis-proneness scales. In GA Miller (Ed), The Behavioral High-Risk Paradigm in Psychopathology. New York: Springer-Verlag.

Endicott J, Spitzer RL, Fleiss JL, Cohen J (1976) The global assessment scale: A procedure for measuring overall severity of psychiatric disturbance. Arch Gen Psychiatry. 33:766-771.

Faraone SV, Green AI, Seidman LJ, Tsuang MT (2001) “Schizotaxia”: Clinical implications and new directions for research. Schizophr Bull. 27:1-18.

Fernandes LOL, Miller GA (1995) Compromised performance and abnormal psychophysiology associated with the Wisconsin psychosis-proneness scales. In GA Miller (Ed), The Behavioral High-Risk Paradigm in Psychopathology. New York: Springer-Verlag.

First MB, Spitzer RL, Gibbon M, Williams JB (1995) The structured clinical interview for DSMIII-R personality disorders (SCID-II). II. Multi-site test-retest reliability study. J Personal Disord. 9:92-104.

Hollingshead AB (1957) Two-Factor Index of Social Position. New Haven: Author.

Keefe RSE, Harvey PD, Lenzenweger MF, Davidson M, Mohs RC, Davis KL (1992) An empirical assessment of the factorial structure of schizophrenic psychopathology: Negative symptoms. Psychiatry Res. 44:153-165.

Kernberg O (1977) The structural diagnosis of borderline personality organization. In P Hartocollis (Ed), Borderline Personality Disorder: The Concept, the Syndrome, the Patient (pp 87-121). New York: International Universities Press.

Kwapil TR (1996) A longitudinal study of drug and alcohol use by psychosis-prone and impulsive-nonconforming subjects. J Abnorm Psychol. 105:114-123. 
Kwapil TR (1998) Social anhedonia as a predictor of the development of schizophrenia spectrum disorders. J Abnorm Psychol. 107:558-565.

Kwapil TR, Chapman LJ, Chapman J (1999) Validity and usefulness of the Wisconsin Manual for rating psychotic-like experiences. Schizophr Bull. 25:363-375.

Kwapil TR, Mann MC, Raulin ML (2002) Psychometric properties and concurrent validity of the schizotypal ambivalence scale. J Nerv Ment Dis. 190:290-295. HTML Full Text

Kwapil TR, Raulin M, Midthun J (2000) A ten-year longitudinal study of intense ambivalence as a predictor of risk for psychopathology. J Nerv Ment Dis. 188:402-408. HTML Full Text

Lenzenweger MF (1998) Schizotypy and schizotypic psychopathology: Mapping an alternative expression of schizophrenia liability. In MF Lenzenweger, RH Dworkin (Eds), Origins and Development of Schizophrenia. Washington, DC: APA Press.

Lenzenweger MF, Dworkin RH (1996) The dimensions of schizophrenia phenomenology: Not one or two, at least three, perhaps four. Br J Psychiatry. 168:432-440.

Lenzenweger MF, Jensen S, Rubin DB (2003) Finding the "genuine” schizotype: A model and method for resolving heterogeneity in performance on laboratory measures in experimental psychopathology research. J Abnorm Psychol. 112:457-468.

Meehl PE (1962) Schizotaxia, schizotypy, schizophrenia. Am Psychol. 17:827-838.

Meehl PE (1964) Manual for Use With Checklist of Schizotypic Signs (No. PR-73-5). Minneapolis: University of Minnesota Research Laboratories of the Department of Psychiatry. 
Meehl PE (1989) Schizotaxia revisited. Arch Gen Psychiatry. 46:935-944.

Meehl PE (1990) Toward an integrated theory of schizotaxia, schizotypy and schizophrenia. J Personal Disord. 4:1-99.

Minsky M (2006) The Emotion Machine: Commonsense Thinking, Artificial Intelligence and the Future of the Human Mind. New York: Simon and Schuster.

Raulin ML (1984) Development of a scale to measure intense ambivalence. J Consult Clin Psychol. 52:63-72.

Raulin ML (1986) Schizotypal Ambivalence Scale. Unpublished test copies available from: ML Raulin, Psychology Department, SUNY at Buffalo, Buffalo, NY 14260.

Raulin ML, Brenner V (1993) Ambivalence. In CG Costello (Ed), Symptoms of Schizophrenia (pp 201-226). New York: Wiley.

World Health Organization (1995) International Personality Disorder Examination Manual DSM-IV Module. New York: Cambridge University Press. 